Portland State University

PDXScholar

$5-1-1970$

\title{
Hot Electron Injection into Liquid Argon from a Tunnel Cathode
}

\author{
Pavel Smejtek \\ Portland State University \\ David G. Onn \\ M. Silver \\ P. Kumbhare
}

Follow this and additional works at: https://pdxscholar.library.pdx.edu/phy_fac

Part of the Physics Commons

Let us know how access to this document benefits you.

\section{Citation Details}

P. Smejtek, M. Silver, Kumbhare, P. and D. G. Onn. Hot Electron Injection into Liquid Argon from a Tunnel Cathode. J. Chem. Phys., 52: 5195-5199, 1970.

This Article is brought to you for free and open access. It has been accepted for inclusion in Physics Faculty Publications and Presentations by an authorized administrator of PDXScholar. Please contact us if we can make this document more accessible: pdxscholar@pdx.edu. 


\title{
Hot Electron Injection into Liquid Argon from a Tunnel Cathode*
}

\author{
M. Silver, P. Kumbhare, P. Smejtek, and D. G. OnN \\ Physics Department, University of North Carolina, Chapel Hill, North Carolina
}

(Received 17 September 1969)

\begin{abstract}
Hot electrons from a tunnel cathode have been injected into liquid argon $\left(99.998 \%\right.$ pure) at $87^{\circ} \mathrm{K}$. The current vs voltage characteristics indicate that the injected hot electrons thermalize very slowly, losing their energy only by elastic scattering processes and finally by capture by the dilute impurities. The deduced thermalization time and distance are very long compared with that in helium, where bubble formation is responsible for energy loss.
\end{abstract}

Over the past few years there has emerged a wealth of information to indicate that the excess negative charge in liquid argon can be characterized as a free or at least a quasifree particle having a small effective mass $^{1,2}\left(m^{*} \simeq m_{0}\right)$. This may be contrasted with the excess negative charge in liquid helium which consists of a self-trapped electron in a bubble of radius $\simeq 15 \AA$. The difference between an electron in liquid argon and liquid helium shows up in the drift mobility. The electron mobility in argon ${ }^{2}$ at $87^{\circ} \mathrm{K}$ is around 500 $\mathrm{cm}^{2} / \mathrm{V} \cdot \mathrm{sec}$, while in helium ${ }^{3}$ at $4.2^{\circ}$ it is around $2 \times 10^{-2}$ $\mathrm{cm}^{2} / \mathrm{V} \cdot \mathrm{sec}$.

The electron-helium interaction is strongly repulsive because of Pauli exclusion. It is this repulsive force which pushes the helium atoms away from the electron to form the bubble. It has been shown ${ }^{4}$ that thermalization and bubble formation is a result of inelastic scattering processes. These inelastic processes result in a very short thermalization time $\left(\sim 10^{-12} \mathrm{sec}\right)$ and indicate a very short $\mathrm{mfp}$ for momentum exchange scattering. On the other hand, one does not have the strong net repulsion in liquid argon, and one might suspect that, in contrast with helium, elastic scattering processes may be involved in the thermalization of the hot electrons and that the mfp for momentum exchange scattering is long as indicated from the mobility measurements. The thermalization time would therefore be relatively long compared to helium, and relatively large efficiency for injection of electrons would be expected even at low applied fields.

We have studied electron injection into liquid argon not only to verify the above predictions, but also to obtain a model for injection for a case where the freeelectron behavior is well understood so that we can use it as a basis for comparison for injection into liquids such as helium where the free-electron properties are more complicated. Interpretation of these data leads us to the conclusion that thermalization is indeed slow. The time may be limited by purely elastic scattering processes or it may be limited by trapping by impurities or both. If it is the former, there are about $10^{5}$ energy exchange scattering events. Using values for the energy and momentum exchange cross section derived by Lekner, ${ }^{2}$ we are able to calculate the magnitude of the current and its dependence upon voltage on the basis of elastic scattering processes and trapping.

Tunnel cathodes have been shown to be very stable sources of hot electrons for emission into liquids. Approximately $10^{-9} \mathrm{~A} / \mathrm{cm}^{2}$ have been stably injected into liquid helium from an $\mathrm{Al}-\mathrm{Al}_{2} \mathrm{O}_{3}-\mathrm{Au}$ structure. We have used the same type junctions to inject electrons into liquid argon. Currents as large as $10^{-9} \mathrm{~A} / \mathrm{cm}^{2}$ have been observed here as well. The detailed use and construction of these diodes have been described elsewher $e^{5}$ and will not be repeated here.

Schematic diagrams of the cryostat and the circuitry used are shown in Figs. 1 and 2. The argon used was obtained from a tank which had a purity of $99.998 \%$. The argon gas was passed through a condenser immersed in liquid nitrogen. The condensed gas was then forced under pressure into the cryostat.

Emission data for the diodes used were taken at liquid-argon temperatures $\left(87^{\circ} \mathrm{K}\right)$. Without warming up the diode, liquid argon was then admitted into the sample chamber. In order to reduce bubbling of the argon in the sample chamber we pumped on the argon in the cryostat and in the chamber, lowering the temperature slightly. All valves were closed afterwards, and no bubbling was observed for periods of up to $30 \mathrm{~min}$. When bubbling reappeared, the pumping procedure was repeated.

The bubbling was a problem because it introduced oscillations in the dark current. No effect on the magnitude of the current observed could be detected by our pumping procedure.

Data on emission into vacuum and argon were usually taken over a period of several days. During this time the diodes were always maintained at $87^{\circ} \mathrm{K}$ and not allowed to warm up. Most of the diodes used had an oxide thickness of around $110 \AA$. Data were also taken on diodes which had an oxide thickness between 110 and $140 \AA$. No major differences could be detected as a function of thickness in this range.

The electrode spacing was generally $0.078 \mathrm{~cm}$ (emitter to collector spacing, see Fig. 2). Data were also taken at 0.178 and $0.5 \mathrm{~cm}$. Some results were also obtained on emission into argon vapor at $87^{\circ} \mathrm{K}$ and atmospheric pressure. 


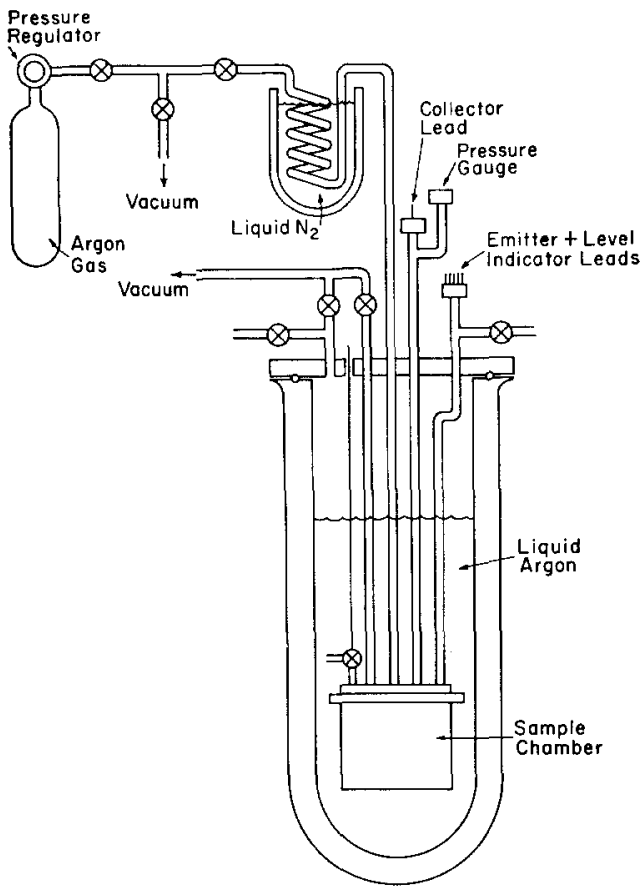

FIG. 1. Schematic diagram of atparatus.

\section{EXPERIMENTAL RESULTS}

Basically the data obtained were the collector current vs the collector voltage.

Similar data were obtained previously on helium. ${ }^{4}$ In the case of helium, because energy relaxation was very fast the electrons had difficulty overcoming their image potential. As a consequence, it was found that the current obeyed the following law which could be derived from the continuity of current equation:

$$
i=\frac{i_{0}}{1+1.5 X_{0} / x_{s}} \exp \left(-\frac{X_{m}}{X_{0}}\right)
$$

where $X_{m}=(e / 4 e E)^{1 / 2}$ is the position of the maximum of the potential, $i_{0}$ is the current available from the electrode, $X_{s}$ is the mean free path for momentum exchange scattering, and $X_{0}=(D \tau)^{1 / 2}$ where $D$ is the diffusion coefficient of the hot electron and $\tau$ is its lifetime for thermalization. This law predicts that a semi$\log$ plot of $i$ vs $X_{m}$ will yield a straight line whose slope is the range of hot electrons. The range in helium was of order $10^{-6} \mathrm{~cm}$.

Our results for argon for three different spacings are given in Fig. 3 along with results into helium and argon vapor for comparison. As can be seen, the semilog plot of the ratio of the current into argon liquid to the current into vacuum vs the collector voltage do not resemble at all the results in helium nor do they yield a straight line. Also notice that at high fields, small $X_{m}$, the current ratios are independent of electrode spacing. There are some slight differences at low fields due to aging effects of the diode, but more importantly, space-charge effects which will be discussed.

The difference between the $i$ vs $V$ characteristics in argon and in helium are also evident when linear scales are used. Figure 4 shows the same data obtained for Fig. 3 plotted linearly in current and voltage. It is apparent from Fig. 4 as well as Fig. 3 that the major difference is in the magnitude of the current at low fields. In argon after an initial rapid rise as a function of field the current does not increase very rapidly, while in helium the current is immeasurable small until quite large fields $(2 \mathrm{kV} / \mathrm{cm})$ and then starts to increase. This behavior is what one would expect for a slowly relaxing electron in argon compared with the fast relaxing electron in helium.

For large electrode spacing, the current in argon is also small at low fields. This is more apparent in Fig. 3 than in Fig. 4. This decrease in current is due to spacecharge limitation. Our argon is only $99.998 \%$ pure; therefore, for moderate fields and the relatively large electrode spacing $(5 \mathrm{~mm})$ the electrons are captured by impurities to form ions. The ion mobility is approximately $6 \times 10^{-4} \mathrm{~cm}^{2} / \mathrm{V} \cdot \mathrm{sec}^{6}{ }^{6}$ At these current levels and small mobilities space-charge limitation will be apparent at field strengths of $10^{3} \mathrm{~V} / \mathrm{cm}$ and less. This is seen in Fig. 5 where the argon data and the theoretical value for the mobility ${ }^{6}$ are plotted on a $\log -\log$ scale. The current could of course never be larger than that indicated by the solid curve representing the theoretical response. These results indicate that the emis-

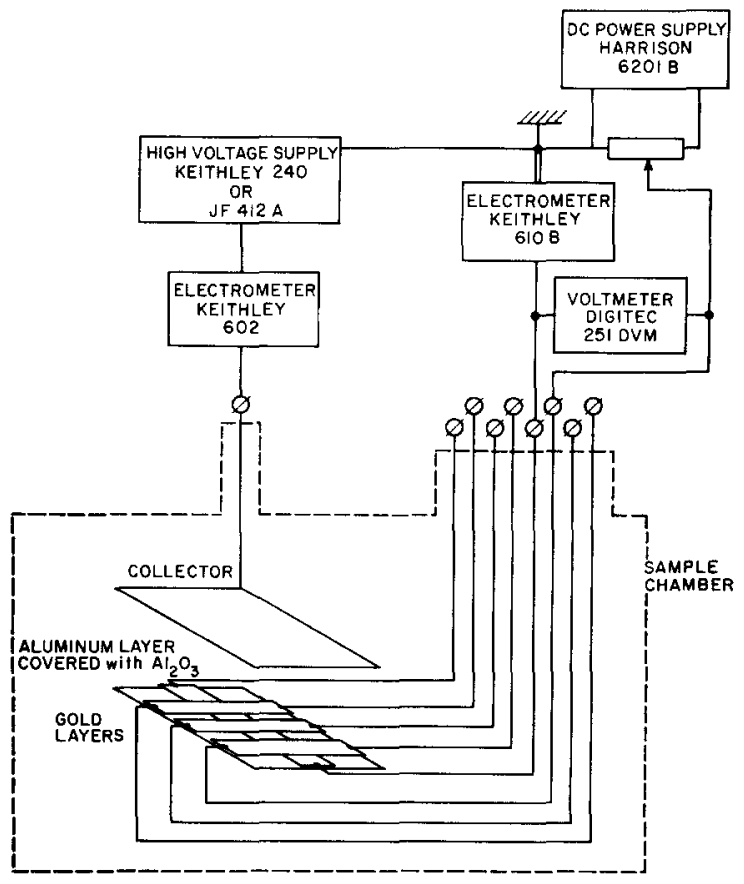

FIG. 2. Schematic representation of the diode along with the associated electronics. 
sion from the electrode is reasonably planar because the area used to calculate the theoretical space-chargelimited current was twice the geometrical area of the emitting surface. (The factor 2 comes in because the spacing is approximately twice the lateral dimension of the emitter.) As pointed out by Rose, ${ }^{7}$ this finite geometry allows a larger current density than the infinite electrode case. The correction is approximately equal to the ratio of the electrode spacing to the radius of the emitting electrode. There is also a lateral selfrepulsion on the electron beam which also causes some spreading of the same order. These results are consistent with space-charge-limited current studies at room temperature in cyclohexane using similar emitters. ${ }^{8}$

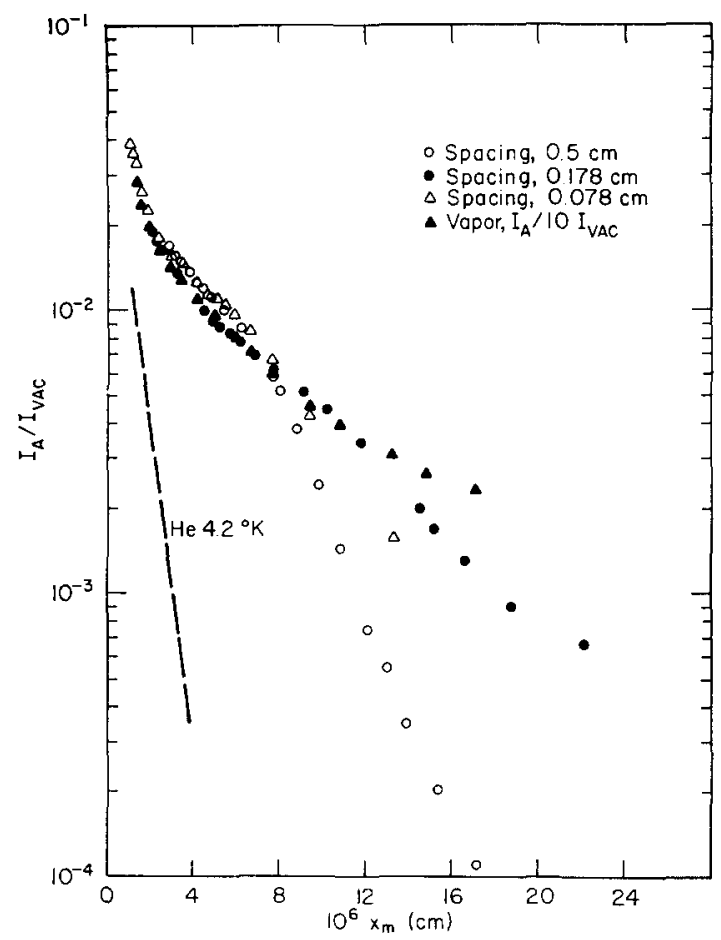

FIG. 3. Semilog plot of the ratio of the current emitted into argon to the current emitted into vacuum vs the position of ithe maximum in the potential.

Also shown in Fig. 5 are the data for $0.78-\mathrm{mm}$ spacing as well as its theoretical space-charge-limited current. As can be seen, the observed current is always well below the space-charge-limited current value.

\section{DISCUSSION}

It is clear from the large magnitude currents observed at low field strengths that the electron is not having difficulty drifting away from the electrode. This implies that the energy relaxation is slow. The energyloss process may be slow because only elastic scattering is involved, because there are inelastic loss processes due only to a dilute concentration of impurities, or

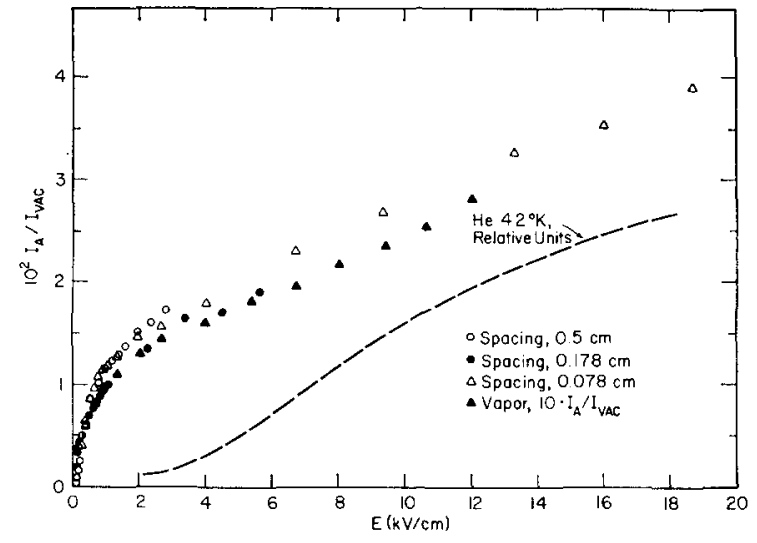

FIG. 4. Linear plot of the ratio of the current emitted into argon to the current emitted into vacuum vs the applied field strength.

because of a combination of both. We know that for large electrode spacing the current is space charge limited and the carriers have a mobility less than $10^{-3}$ $\mathrm{crn}^{2} / \mathrm{V} \cdot \mathrm{sec}$ consistent with the value for an impurity negative ion. ${ }^{6}$

If we assume a very simple model based on elastic scattering processes thermalizing the injected hot electrons to an energy determined by the electric field and subsequent capture by impurities, we obtain agreement with experimental results. This is essentially a two-

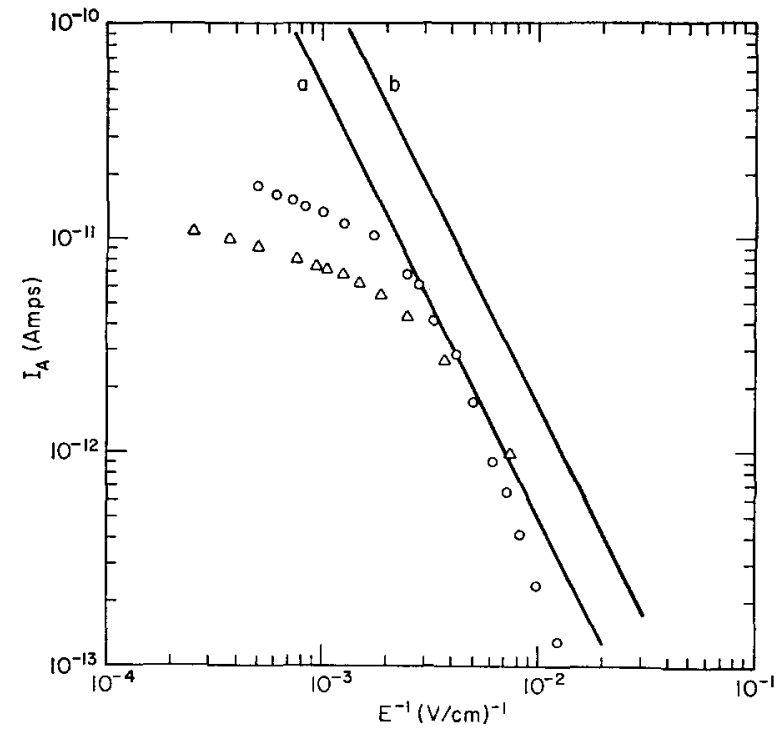

FIG. 5. Log-log plot of the current emitted into argon vs the reciprocal of the applied field strength. Solid curves $a$ and $b$ are the theoretical space-charge-limited currents for 5-mm spacing and $0.78-\mathrm{mm}$ spacing, respectively. The open circles are the experimental data for 5-mm spacing, and the triangles represent the $0.78-\mathrm{mm}$ spacing. The difference between the high field currents for the two spacings is a result of the difference in efficiency between the two different diodes used. The diode used with the large spacing happened to be more efficient. Also, the current at very low applied field is less than expected because of the effect of the fringe field of the diode. 


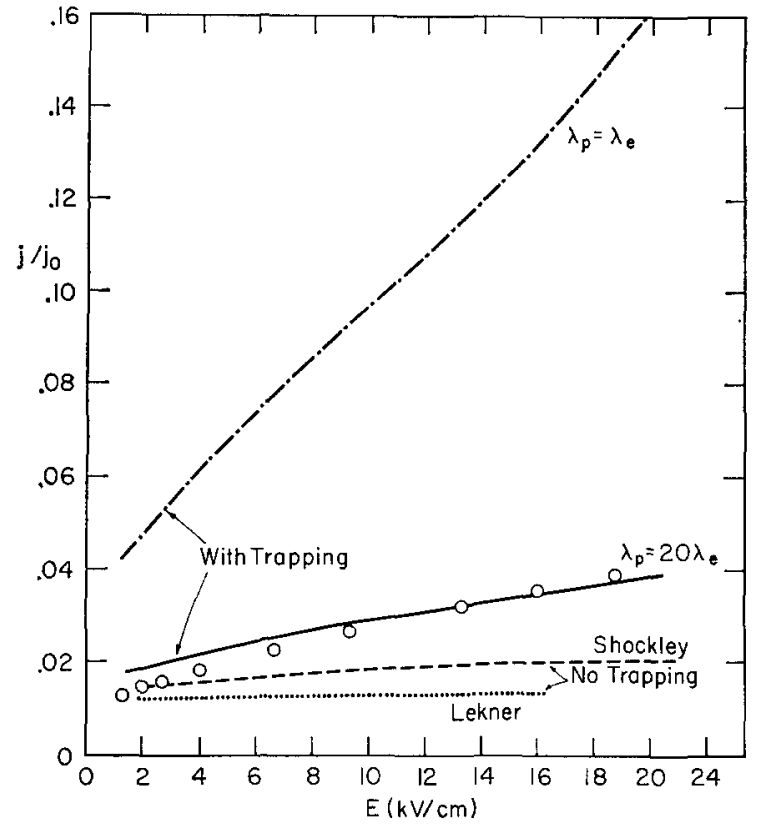

FIG. 6. The theoretical shape of the ratio of the current to the magnitude of the available current vs field strength with and without trapping by impurities is shown. Also shown are the theoretical curves considering equal mfp for momentum and energy exchange scattering and for 20 times different mfp as calculated by Lekner. The experimental results are also shown by the circles.

state model for the free electron and is obviously an oversimplification. At present random walk calculations are being attempted which should give a more complete picture. The model involves solving the continuity of current equation and identifying three different carriers: $n_{1}$, the density of injected hot electrons; $n_{2}$, the density of quasifree electrons in equilibrium with the field; and $n_{3}$, the density of impurity ions.

The three equations to solve are

$$
\begin{aligned}
-\nabla \cdot J_{1}-k_{1} n_{1} & =0, \\
-\nabla \cdot J_{2}+k_{1} n_{1}-k_{2} n_{2} & =0, \\
-\nabla \cdot J_{3}+k_{2} n_{2} & =0,
\end{aligned}
$$

where $k_{1}$ and $k_{2}$ are the relaxation rate constants of $n_{1}$ and $n_{2}$, respectively.

The boundary condition for injected hot electrons in terms of the available current is

$$
\left.n_{1}\right|_{x=0}=\frac{j_{i}}{\left(v_{i} / 4+k / \gamma_{1}\right)},
$$

where $v_{i}$ is the random velocity of the injected hot electrons, $j_{i}$ is the current available from the electrode, and $\gamma_{1}$ is the exponent of the exponential solution to Eq. (1). To solve these equations for the current we assume a constant field. Such an assumption is reasonable for slowly relaxing electrons because the diffusion length will be large compared with the range of any image field potential which is $5 \times 10^{-6} \mathrm{~cm}$ or less for the applied fields used. In an Appendix, we show the calculation for the current $j$, which is

$$
\begin{array}{r}
\left.\left.j=j_{i}\left(\gamma_{1} / x_{2}{ }^{2}\right)\left[1+1.5 \gamma_{1} x_{1}^{2} / \lambda_{p}\right)\right]^{-1}\left[\left(x_{2}^{-2}\right)^{-1}\right)-\beta \gamma_{1}-\gamma_{1}^{2}\right]^{-1} \\
{\left[\gamma_{1}^{-1}\left(1+\gamma_{1} / \delta\right)^{-1}-\alpha^{-1}(1+\alpha / \delta)^{-1}\right] .}
\end{array}
$$

The other symbols are as follows: $x_{2}^{2}=D_{2} / k_{2}, x_{1}{ }^{2}=D_{1} / k_{1}$, $\beta=\mu_{2} E / D_{2}, \lambda_{p}$ is the mean free path for momentum exchange collision,

$$
\alpha=-\frac{1}{2} \beta+\left(\frac{1}{4} \beta^{2}+1 / x_{2}^{2}\right),
$$

and $\delta=D_{3} / \mu_{3} E$. The D's, $\mu$ 's, and $E$ are the diffusion constants, mobilities, and the applied electric field strength. The magnitude of $x_{1}$ and $x_{2}$ are

$$
x_{1}=\lambda_{p}\left[(M / 2 m)\left(\lambda_{e} / 6 \lambda_{p}\right) \ln \left(\epsilon_{i} / \epsilon_{2}\right)\right]^{1 / 2},
$$

where $\lambda_{e}$ is the mean free path for energy exchange collisions and

$$
x_{2}=\left(\lambda_{p} / 6 n_{i} \sigma_{i}\right)^{1 / 2},
$$

where $n_{i}$ is the impurity concentration and $\sigma_{i}$ is the electron capture cross section of the impurity. The values of $\lambda_{p}$ and $\lambda_{e}$ are given in the paper by Lekner ${ }^{2}$ and are approximately $1.4 \times 10^{-6}$ and $7 \times 10^{-8} \mathrm{~cm}$, respectively. The values of $n_{i}$ were obtained from the known impurity concentration of the tank gas (20 $\mathrm{ppm}), \sigma_{i}$ was assumed to be that of an uncharged molecule $\left(\sim 10^{-15} \mathrm{~cm}^{2}\right)$, and $\gamma_{1}$ was calculated using a mfp obtained from Lekner. ${ }^{2}$ The final energy of the $n_{2}$ electrons as a function of field strength is also given in the paper by Lekner. ${ }^{2}$ We therefore estimate $x_{1} \approx$ $5 \times 10^{-5}$ and $x_{2} \approx 2 \times 10^{-5}$ from Eqs. (6) and (7), both of which are large compared with the range of the image potential.

We have all the information needed to calculate $j$ vs $E$ assuming only that the average energy of the injected hot electron is $1 \mathrm{eV}$. (This is a reasonable value based on our measurements of the velocity distribution of hot electrons emitted into vacuum.)

Figure 6 shows a plot of $j$ vs $E$ from Eq. (5) using the Lekner values for the $\lambda$ 's. As pointed out by Lekner, ${ }^{2}$ the difference between $\lambda_{p}$ and $\lambda_{e}$ is due to the structure factor. We have also calculated $j$ vs $E$ assuming $\lambda_{p}=\lambda_{e}$ and using the value given by Lekner for $\lambda_{e}$ which does not include the structure factor. Very reasonable agreement is obtained from this theory when $\lambda_{p}=20 \lambda_{e}$, but agreement is poor for $\lambda_{p}=\lambda_{e}$. This is not unexpected considering the agreement between the theory of drift velocity and the experimental results of Spear. ${ }^{1}$

It is interesting to compare the theoretically expected current in very pure argon with the present experimental results. A theoretical value of 0.013 is obtained when one uses Lekner's value for the steady-state energy of the electrons vs electric field in the region $10^{3}-2 \times 10^{4} \mathrm{~V} / \mathrm{cm}$. The magnitude of the current is almost independent of electric field. On the other hand, 
when one uses the Shockley theory ${ }^{9}$ one obtains a value of $0.013-0.021$ in the same field strength region. Our present results compare very favorably to the Shockley predictions, and one would be tempted to consider them except for the known trapping at impurities. In either case, impurities are expected to have less than a 2:1 effect on the injection current. Further, impurities in the part per million range increase the current because they inhibit back diffusion.

Recently, Halpern et al..$^{10}$ photoinjected electrons into argon in order to determine the electron affinity of this liquid. They made no correction for back diffusion as a function of the energy of the hot electron in argon, nor did they correct for the escape cone into vacuum. Without these corrections, their value for the electron affinity of 0.33 is in doubt.

\section{APPENDIX}

Equation (5) of the text is derived from Eqs. (1)-(3) when one assumes a constant field. In fact the field is not constant near the electrode because of the distortion due to the image potential. The range of this distortion is either $(e / 4 \epsilon E)^{1 / 2}$ or $\left(e^{2} / 4 \epsilon k T\right)$, whichever is less. For our range of applied fields, the first term has a value between $5 \times 10^{-6}$ and $10^{-6}$ while the second term has a value of around $4 \times 10^{-6}$. Therefore, if the distance traveled during thermalization or trapping by impurities is long compared with the range of the image potential, then the constant field approximation is reasonable. The derivation is as follows:

$$
-D_{1} d^{2} n_{1} / d x^{2}+\mu_{1} E d n_{1} / d x+k_{1} n_{1}=0 .
$$

Using the boundary condition given by Eq. (4), the solution to (A1) is

where

$$
n_{1}=j_{i}\left(\frac{1}{4} v_{i}+k_{1} / \gamma_{1}\right)^{-1} \exp \left(-\gamma_{1} x\right)
$$

$$
\gamma_{1}=-\mu_{1} E / 2 D_{1}+\left[\left(\mu_{1} E / 2 D_{1}\right)+k_{1} / D_{1}\right]^{1 / 2} .
$$

(Notice that for very small fields $\gamma_{1}$ is the reciprocal of the diffusion length of the injected electrons during the time $k_{1}^{-1}$.)

The continuity equation for the quasifree electrons in equilibrium with the electric field becomes from Eqs. (2) and (A2),

$$
\frac{d^{2} n_{2}}{d x^{2}}-\frac{\mu_{2} E}{D_{2}} \frac{d n_{2}}{d x}-\frac{k_{2}}{D_{2}} n_{2}=\frac{k_{1}}{D_{2}} j_{i}\left(\frac{v_{i}}{4}+\frac{k_{i}}{\gamma_{1}}\right)^{-1} \exp \left(-\gamma_{1} x\right)
$$

The solution to (A4) is

$$
\begin{aligned}
n_{2}=j_{i}\left(1 / x_{2}^{2}\right)\left[\frac{1}{4} v_{i}+\right. & \left.k_{1} / \gamma_{1}\right]^{-1}\left[1 / x_{2}^{2}-\beta \gamma_{1}-\gamma_{1}^{2}\right]^{-1} \\
& \times\left[\exp \left(-\gamma_{1} x\right)-\exp (-\alpha x)\right],
\end{aligned}
$$

assuming $\left.n_{2}\right|_{x=0}=0$.

Equation (3) can now be rewritten as

$$
d\left(J_{3}+k_{2} \int n_{2} d x\right) / d x=0,
$$

or the collected current $j$ is

$$
j=-\frac{D_{3} d n_{3}}{d x}+\mu_{3} n_{3} E+k_{2} \int_{0}^{\pi} n_{2} d x
$$

From the boundary condition that $\left.n_{3}\right|_{x=0}$ and $\left.n_{3}\right|_{x=l}=0$ ( $l$ is the thickness of the sample),

$$
\begin{aligned}
j=\frac{j_{i}}{x_{2}^{2}} k_{1}\left(\frac{1}{4} v_{i}\right. & \left.+\frac{k_{1}}{\gamma_{1}}\right)^{-1}\left(\frac{1}{x_{2}^{2}}-\beta \gamma_{1}-\gamma_{1}^{2}\right)^{-1} \\
& \times\left[\gamma_{1}^{-1}\left(1+\frac{\gamma_{1}}{\delta}\right)^{-1}-\alpha^{-1}\left(1+\frac{\alpha}{\delta}\right)^{-1}\right] .
\end{aligned}
$$

Finally, because $D_{1}=v_{i} \lambda_{p} / 6$,

$$
k_{1}\left(\frac{1}{4}+k_{1} / \gamma_{1}\right)^{-1}=\gamma_{1}\left(1+1.5 \gamma_{1} x_{1}^{2} / \lambda_{p}\right)^{-1},
$$

and therefore

$$
\begin{aligned}
j=j_{i} \frac{\gamma_{1}}{x_{2}{ }^{2}}\left(1+\frac{1.5 \gamma_{1} x_{1}^{2}}{\lambda_{p}}\right)^{-1}\left(\frac{1}{x_{2}^{2}}-\beta \gamma_{1}-\gamma_{1}\right)^{-1} \\
\times\left[\gamma_{1}^{-1}\left(1+\frac{\gamma_{1}}{\delta}\right)^{-1}-\alpha^{-1}\left(1-\frac{\alpha}{\delta}\right)^{-1}\right] .
\end{aligned}
$$

* Supported in part by the National Science Foundation, the University of North Carolina Materials Research Center through Contract SD-100, and the Army Research Office-Durham.

${ }^{1}$ L. S. Miller, S. Howe, and W. E. Spear, Phys. Rev. 166, 871 (1968).

${ }^{2}$ J. Lekner, Phys. Rev. 158, 130 (1967).

${ }^{3}$ L. Meyer, H. T. Davis, S. A. Rice, and R. J. Donnelly, Phys. Rev. 126, 1927 (1962).

${ }^{4}$ D. G. Onn and M. Silver, Phys. Rev. 183, 295 (1969).

${ }^{5}$ C. R. Crowell and S. M. Sze, Physics of Thin Films, edited by G. Haas and R. E. Thun (Academic, New York, 1967), Vol. 4, p. 325 .

${ }^{6}$ H. T. Davis, S. A. Rice, and L. Meyer, J. Chem. Phys. 37, 2470 (1962).

${ }^{7}$ We are indebted to $\mathrm{A}$. Rose for pointing out this correction for finite geometry.

${ }^{8}$ M. Silver, D. G. Onn, and P. Smejtek, J. Appl. Phys. 40, 2222 (1969).

${ }^{9}$ W. Shockley, Bell System Tech. J. 30, 990 (1951).

${ }^{10}$ B. Halpern, J. Lekner, S. A. Rice, and R. Gomer, Phys. Rev. 156, 351 (1967). 